\title{
Adubação com boro em batata-doce: efeito das fontes, doses e modos de aplicação
}

\section{Boron fertilization on sweet potato: effect of sources, rates and application form}

\author{
Fábio Rafael Echer ${ }^{1 *}$; José Eduardo Creste ${ }^{2}$
}

\begin{abstract}
Resumo
A adubação boratada pode proporcionar aumentos na produtividade e na qualidade das raízes tuberosas de batata-doce, no entanto poucos trabalhos contemplam esse assunto. Objetivou-se avaliar a produtividade da cultura da batata-doce em resposta a fontes, doses e modos de aplicação de B. O delineamento experimental utilizado foi o de blocos casualizados, em esquema fatorial $((2 \times 2 \times 2)+1)$, com três repetições. Os tratamentos foram aplicados em cobertura, sendo resultados da combinação de fontes de B (ácido bórico e bórax), formas de aplicação (via solo e via foliar) e doses de B (1 e $\left.2 \mathrm{~kg} \mathrm{ha}^{-1}\right)$ mais uma testemunha. Não houve interação entre os fatores estudados sobre a produtividade comercial de batata-doce. As formas de aplicação, via solo e via foliar bem como as fontes ácido bórico e bórax não apresentaram diferenças entre si, mas proporcionaram produtividades superiores ao tratamento sem $\mathrm{B}$. A produtividade de batata-doce foi favorecida com a aplicação de $2 \mathrm{~kg} \mathrm{ha}^{-1}$ de B.
\end{abstract}

Palavras-chave: Ipomoea batatas, bórax, ácido bórico, produtividade comercial

\begin{abstract}
Boron (B) fertilization can result in high yield and better sweet potato storage roots quality. However, there is few works about this subject. The objective was to evaluate the yield of sweet potato crops in response to B sources, rates and application form. The experimental design was complete randomized blocks, in factorial outline $((2 \times 2 \times 2)+1)$, with three replications, which resulted of the combination of sources of B (boric acid and borax), application form (via soil and via foliar) and doses of B (1 and 2 $\mathrm{kg} \mathrm{ha}^{-1}$ ) and a control treatment (without B). There was no interaction between the factors studied for the commercial yield of sweet potato. No significant differences was found between B sources and application form, but they showed higher yield than the control treatment. Additionally, sweet potato yield increade with application of $2 \mathrm{~kg} \mathrm{ha}^{-1}$ of $\mathrm{B}$.
\end{abstract}

Key words: Ipomoea batatas, borax, boric acid, commercial productivity

$\mathrm{O}$ fornecimento de $\mathrm{B}$ às culturas têm principalmente para os consumidores brasileiros de proporcionado aumentos em suas produtividades baixa renda, sendo a batata-doce uma importante (BRITO NETO et al., 2011; BYJU; fonte de B para a nutrição humana (GAD; CANDIL, NEDUNCHEZHIYAN; NASKAR, 2007). As 2008). Cada grama de batata-doce contém raízes tuberosas de batata-doce constituem a fonte aproximadamente $1,08 \mu \mathrm{g}$ de $\mathrm{B}$, e a exportação pode mais popular de energia, minerais e vitaminas, alcançar 1,32 kg de B para produtividades da ordem

\footnotetext{
1 Eng ${ }^{\circ}$ Agr $^{\circ}$, Doutorando em Agricultura, Universidade Estadual Paulista, FCA/UNESP. Departamento Produção Vegetal, Rua José Barbosa de Barros, 1780. Cx Postal 237, Botucatu-SP, CEP: 18610-307. E-mail: fabioecher@fca.unesp.br

2 Pró-Reitor Acadêmico da Universidade do Oeste Paulista, UNOESTE, Campus I, Presidente Prudente, SP. E-mail: jcreste@ unoeste.br

* Autor para correspondência
} 
de 20 toneladas por hectare, que são comuns nessa cultura (BYJU; NEDUNCHEZHIYAN; NASKAR, 2007). De acordo com Echer, Dominato e Creste (2009a), para uma produção de 15,4 t ha $^{-1}$ de massa seca total da planta, a extração foi de $0,378 \mathrm{~kg}$ de B.

Os sintomas da deficiência de B em batata-doce caracterizam-se pelo engrossamento e enrolamento de folhas novas, crescimento compacto devido à redução dos internódios, redução de lóbulos e margens irregulares, morte do meristema apical, formação de raízes curtas, grossas e de tubérculos rachados com bolhas em sua superfície (O'SULLIVAN; ASHER; BLAMEY, 1997), pois o B participa da formação da parede celular. A deficiência de $\mathrm{B}$ provoca o acúmulo de ribulose5-P, favorecendo a formação e o acúmulo de fenóis, acarretando em necrose do tecido.

No Brasil, poucos são os trabalhos de pesquisa sobre a adubação de B em batata-doce, mas em alguns manuais de adubação, verifica-se a inclusão da adubação boratada via solo, cujas doses variam de 0,5 a $2 \mathrm{~kg} \mathrm{ha}^{-1}$ de B, (SILVA; LOPES; MAGALHÃES, 2002; REIS; SOUZA; CECÍLIO FILHO, 1996), no entanto em outros manuais de adubação a adubação boratada para a batata-doce não é contemplada (MONTEIRO; PERESSIN, 1997). O declínio da produtividade de batata-doce na Papua Nova Guiné foi associado, entre outros fatores, à fertilidade dos solos, sendo que os baixos teores de $\mathrm{B}$ contribuíram para isso (HARTEMINK et al., 2000).

$\mathrm{Na}$ Austrália a recomendação de $\mathrm{B}$ na cultura da batata-doce varia de 1,0 a $1,5 \mathrm{~kg} \mathrm{ha}^{-1}$ de $\mathrm{B}$ em solos arenosos e ácidos, e até 4,0 $\mathrm{kg} \mathrm{ha}^{-1}$ de $\mathrm{B}$ em solos argilosos e alcalinos, devido principalmente a menor disponibilidade de $\mathrm{B}$ em $\mathrm{pH}$ elevado (O'SULLIVAN; ASHER; BLAMEY, 1997), e nos EUA é recomendada a dose $1,12 \mathrm{~kg} \mathrm{ha}^{-1}$ de B (GRANBERRY; KELLEY; BOYHAN, 2007)

A adubação foliar de B é recomendada para várias culturas, pois permite o uso de doses mais baixas. Em batata-doce, não está claro se a aplicação foliar é efetiva, devido a baixa mobilidade do B (O’SULLIVAN; ASHER; BLAMEY, 1997). Dentre as fontes de B disponíveis no Brasil, destacam-se o bórax (110 $\mathrm{g} \mathrm{kg}^{-1}$ de B) e o ácido bórico (170 g $\mathrm{kg}^{-1} \mathrm{de} \mathrm{B}$ ), que diferem na sua solubilidade. $\mathrm{O}$ bórax possui solubilidade de $21 \mathrm{~g} \mathrm{~L}^{-1}$ enquanto o ácido bórico de $63,5 \mathrm{~g} \mathrm{~L}^{-1}$. No presente estudo, teve-se por objetivo avaliar o efeito de fontes, doses e modos de aplicação de B na produtividade comercial de raízes tuberosas da cultura da batata-doce.

$\mathrm{O}$ experimento foi conduzido entre os meses de agosto de 2007 e janeiro de 2008, em lavoura comercial de batata doce, em Presidente Prudente - SP, definida pelas seguintes coordenadas geográficas: latitude $22^{\circ} 06^{\prime} 50^{\prime} \mathrm{S}$ e longitude $51^{\circ} 27^{\prime} 32$ ' W, à uma altitude média de $406 \mathrm{~m}$, e a classificação climática segundo Koppen é do tipo Aw, tropical chuvoso com inverso seco.

O solo da área foi classificado como Argissolo Vermelho-amarelo arenoso, com as seguintes características na camada de $0-20 \mathrm{~cm}: \mathrm{pH}\left(\mathrm{CaCl}_{2}\right.$ $\left.0,01 \mathrm{~mol} \mathrm{~L}^{-1}\right)$ 5,4; $10 \mathrm{~g} \mathrm{dm}^{-3}$ de $\mathrm{MO} ; 39 \mathrm{mg} \mathrm{dm}^{-3} \mathrm{de} \mathrm{P}$ resina; $15 \mathrm{mmol}_{\mathrm{c}} \mathrm{dm}^{-3} \mathrm{de} \mathrm{H}+\mathrm{Al} ; 2,1 \mathrm{mmol}_{\mathrm{c}} \mathrm{dm}^{-3} \mathrm{de} \mathrm{K}$; $19 \mathrm{mmol}_{\mathrm{c}} \mathrm{dm}^{-3}$ de Ca; $5 \mathrm{mmol}_{\mathrm{c}} \mathrm{dm}^{-3}$ de $\mathrm{Mg} ; 5,6 \mathrm{mg}$ $\mathrm{dm}^{-3}$ de S; 26 mmol $_{\mathrm{c}} \mathrm{dm}^{-3}$ de SB; $41 \mathrm{mmol}_{\mathrm{c}} \mathrm{dm}^{-3} \mathrm{de}$ CTC; saturação por bases de $63 \% ; 40,4 \mathrm{mg} \mathrm{dm}^{-3} \mathrm{de}$ $\mathrm{Mn} ; 19 \mathrm{mg} \mathrm{dm}^{-3}$ de Fe; 0,7 mg dm ${ }^{-3}$ de Cu; 1,9 mg $\mathrm{dm}^{-3}$ de $\mathrm{Zn}$ e $0,13 \mathrm{mg} \mathrm{dm}^{-3}$ de B.

Antes do plantio, o preparo de solo deu-se com duas arações pesadas, três gradagens leves e com a marcação de curvas de níveis. Os sulcos de plantio foram demarcados com espaçamento entre si de $0,90 \mathrm{~m}$. O plantio foi realizado em meados de agosto de 2007, utilizando-se ramas da variedade Canadense, coletadas na mesma propriedade. A adubação mineral de plantio foi de $250 \mathrm{~kg} \mathrm{ha}^{-1}$ do formulado NPK 4-30-10. A adubação mineral de cobertura constou da aplicação de $40 \mathrm{~kg} \mathrm{ha}^{-1}$ de $\mathrm{K}_{2} \mathrm{O}$ na fonte cloreto de potássio, aplicada aos 45 dias após o plantio.

O delineamento experimental utilizado foi o de blocos casualizados, em esquema fatorial 
$((2 \times 2 \times 2)+1)$, com três repetições. Os tratamentos foram aplicados em cobertura, sendo resultados da combinação de fontes de B (ácido bórico e bórax), formas de aplicação (via solo e via foliar) e doses de B (1 e $2 \mathrm{~kg} \mathrm{ha}^{-1}$ ) mais uma testemunha sem B. Cada parcela continha 50 plantas, em uma área de $18 \mathrm{~m}^{2}$, com dimensões de $5 \mathrm{~m} \mathrm{x} \mathrm{3,6} \mathrm{m,} \mathrm{com} \mathrm{quatro} \mathrm{linhas}$ de plantio. A área útil compreendeu as duas linhas centrais da parcela, totalizando $9 \mathrm{~m}^{2}$.

Os tratamentos foram aplicados em cobertura, 60 dias após o plantio. A aplicação foliar nos tratamentos foi realizada com a utilização de um equipamento de pulverização manual de precisão, pressurizado a $\mathrm{CO}_{2}$, com pressão constante de 150 $\mathrm{kPa}$, com bico jato plano do tipo leque, posicionado a aproximadamente $0,5 \mathrm{~m}$ acima do dossel das plantas, com velocidade média de deslocamento de $1,0 \mathrm{~m} \mathrm{~s}^{-1}$ e volume de calda de $500 \mathrm{~L} \mathrm{ha}^{-1}$, determinados em ensaios de calibração preliminares. Para a adubação via solo, misturou-se os adubos com areia em virtude da baixa quantidade de fertilizante, e essa mistura foi aplicada sobre os sulcos de plantio.

A colheita foi realizada em janeiro de 2008, 145 dias após o plantio. A produtividade comercial correspondeu ao peso das raízes de formato uniforme e lisas, classificadas como Especial, Extra B e Extra A, com peso entre 151- $400 \mathrm{~g}$, conforme descrito por Silva, Lopes e Magalhães (2002). O estudo estatístico constou da análise de variância, e para os efeitos significativos as médias foram comparadas pelo teste $\mathrm{t}$ de Student, a 5\% de probabilidade.

Não houve interação significativa entre fontes $\mathrm{x}$ formas de aplicação $\mathrm{x}$ doses de $\mathrm{B}$ sobre a produtividade comercial de batata doce, mas os fatores apresentaram efeitos isolados. Os tratamentos com fontes de B não apresentaram diferenças entre si, no entanto foram mais produtivos que a testemunha sem boro (Figura 1a). Os resultados observados no presente trabalho divergem dos resultados obtidos por Marchetti et al. (2001) em girassol, onde a fonte bórax foi mais eficiente no fornecimento de $\mathrm{B}$ às plantas, no entanto para Mandal, Roy e Mazumdar (2009) a maior produtividade de mostarda foi obtida com a fonte Calbor ${ }^{\circledR}(1 \%$ de B) em relação ao bórax. Esses resultados indicam que as culturas respondem de forma diferenciada á aplicação de fontes de B, pois devido as diferentes solubilidades das fontes, a velocidade de absorção também é diferente.

Figura 1. (a) Produtividade comercial de batata-doce em função das fontes, (b) das formas de aplicação e (c) das doses de B. (a $>$ b pelo teste $t$ de Student (p<0,05); Dms: 3,10 Mg ha ${ }^{-1}$. CV: 12,48\%).

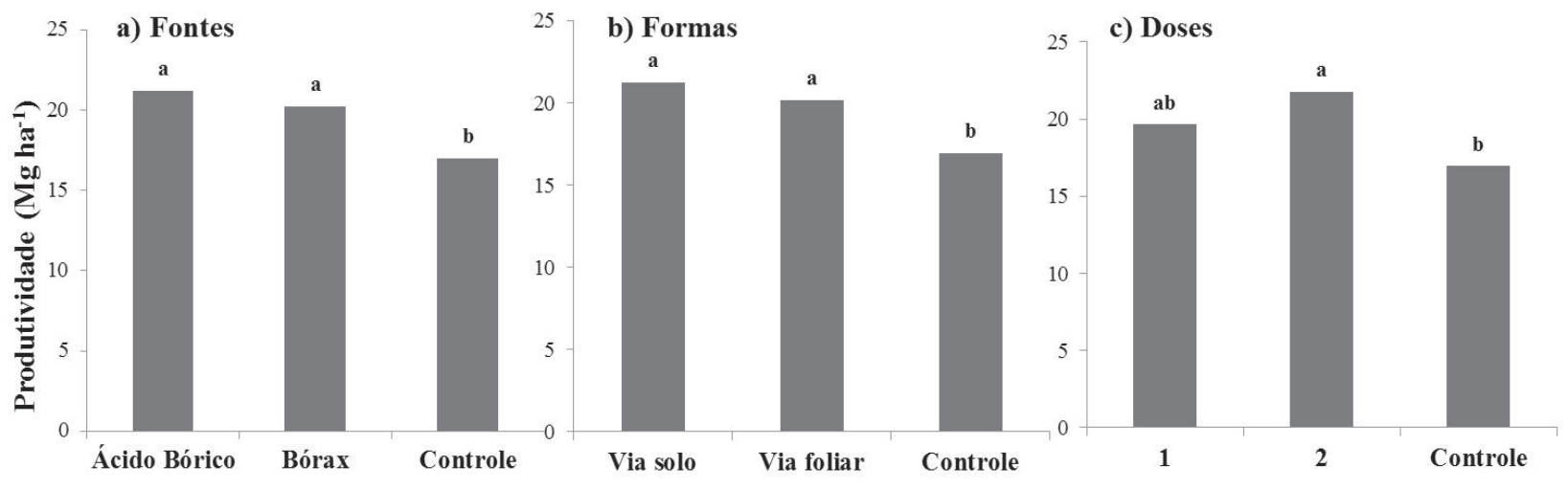

Quanto ás formas de aplicação de B, também não houve diferença significativa entre a aplicação via solo ou via foliar, mas ambos os tratamentos proporcionaram aumentos em produtividade em relação ao tratamento controle (Figura 1b). Tal fato está associado á capacidade das plantas absorverem 
o boro tanto pelas raízes quanto pelas folhas. O mesmo foi observado por Hopkins et al. (2007) que avaliaram a produção da batata inglesa cultivada em solos com altos teores de B no solo, onde se verificou que não houve diferença significativa para o fornecimento de B via solo ou foliar.

Para as doses, o tratamento com $2 \mathrm{~kg} \mathrm{ha}^{-1}$ proporcionou incrementos de produtividade de batata-doce da ordem de $28 \%$ em relação ao tratamento controle, que não recebeu a aplicação de $B$, mas não diferenciou-se estatisticamente do tratamento que recebeu $1 \mathrm{~kg} \mathrm{ha}^{-1}$ de B (Figura 1c).

Essa resposta a aplicação de doses crescentes de B foi associada ao teor de B no solo do presente trabalho, que foi de $0,13 \mathrm{mg} \mathrm{dm}^{-3}$, considerado baixo. Os resultados encontrados condizem com a recomendação de Silva, Lopes e Magalhães (2002), que indicaram a aplicação ao solo de 1 a $2 \mathrm{~kg} \mathrm{ha}^{-1}$ de $\mathrm{B}$ em solos com baixa fertilidade, como é o caso dos solos da região do cerrado brasileiro, no entanto diferem da recomendação indicada por O'Sullivan, Asher e Blamey (1997), Granberry, Kelley e Boyhan (2007) e por Reis, Souza e Cecílio Filho (1996) que indicaram a utilização de doses que variam de 0,5 a 1,5 $\mathrm{kg} \mathrm{ha}^{-1}$ de B em solos com baixos teores desse micronutriente. Em trabalho que avaliou a interação ente doses de K e B, Echer et al. (2009b) observaram que a aplicação de um potencializou a ação do outro, e a produtividade máxima de raízes tuberosas foi obtida com a aplicação de $2 \mathrm{~kg} \mathrm{ha}^{-1}$ de B juntamente com $200 \mathrm{~kg} \mathrm{ha}^{-1}$ de $\mathrm{K}$, dose de B essa que também proporcionou a maior produtividade no presente trabalho.

Byju, Nedunchezhiyan e Naskar (2007) avaliaram a resposta à aplicação de diferentes doses de B em batata-doce, cultivada em solos da Índia, com teores de B no solo da ordem de $0,35 \mathrm{mg} \mathrm{dm}^{-3}$, e observaram que as maiores produtividades obtidas nos anos de 2001-2002; 2002-2003 e 2003-2004 foram de 16,$11 ; 18,14$ e $17,83 \mathrm{Mg} \mathrm{ha}^{-1}$ com a dose de $1,5 \mathrm{~kg} \mathrm{ha}^{-1}$ de B. É preciso salientar que o teor de $\mathrm{B}$ no solo do presente experimento foi considerado baixo $\left(0,13 \mathrm{mg} \mathrm{dm}^{-3}\right)$, sendo assim, este apresentou alta resposta á aplicação de B.

Em trabalho com doses de B aplicado ao solo na cultura da batata inglesa, Mesquita et al. (2007) observaram que a produção de tubérculos dos cultivares Asterix e Monalisa foi diferenciada quando cultivadas em Latossolos e em Cambissolos em resposta ao aumento da dose de B. A produtividade de ambos os cultivares foi maior no Latossolo (teor de B no solo de 0,4 $\mathrm{mg} \mathrm{dm}^{-3}$ ) nas doses de 2,19 e de $1,59 \mathrm{mg} \mathrm{dm}^{-3}$ para os cultivares Asterix e Monalisa, respectivamente, sendo que os autores atribuíram á resposta ao boro aplicado em virtude dos baixos teores de $\mathrm{B}$ nos solos estudados. Também na cultura da batata inglesa, Pregno e Armour (1992) obtiveram aumentos em produtividade de 19,7 para 27,3 $\mathrm{Mg}$ ha $^{-1}$ com a aplicação de 2,0 $\mathrm{kg} \mathrm{ha}^{-1}$ de $\mathrm{B}$, mostrando assim que espécies que possuem raízes de armazenamento ou tubérculos apresentam melhorias na produtividade em razão da aplicação de B.

Em uma condição de maior fertilidade do solo em relação ao B do que no presente trabalho, Hopkins et al. (2007) avaliaram a resposta da cultura da batata inglesa á doses de B em solos de textura média, com teores de B no solo da ordem de $0,57 \mathrm{mg} \mathrm{dm}^{-3}$, e concluíram que nessa situação, não houve diferença significativa das doses de $\mathrm{B}$ aplicadas até $3,3 \mathrm{~kg}$ $\mathrm{ha}^{-1}$, mostrando assim, que a resposta das culturas á aplicação de $\mathrm{B}$ depende, dentre outros fatores, do tipo de solo, do teor do nutriente no solo e da exigência da cultura.

Não há diferenças entre as fontes e entre as formas de aplicação de $\mathrm{B}$, sendo todas mais produtivas que a testemunha. A produtividade de batata-doce é maior quando é utilizado $2 \mathrm{~kg} \mathrm{ha}^{-1}$ de B.

\section{Referências}

BRITO NETO, J. F.; PEREIRA, W. E.; CAVALCANTI, L. F.; ARAÚJO, R. C.; LACERDA, J. S. Produtividade e qualidade de frutos de mamoeiro 'sunrise solo' em função de doses de nitrogênio e boro. Semina: Ciências 
Agrárias, Londrina, v. 32, n. 1, p. 69-80, jan./mar. 2011.

BYJU, G.; NEDUNCHEZHIYAN, M.; NASKAR, S. K. Sweet potato response to boron application on an Alfisols in the subhumid tropical climate of India. Communications in Soil Science and Plant Analysis, New York, v. 38, n. 17/18, p. 2347-2356, 2007.

ECHER, F. R.; DOMINATO, J. C.; CRESTE, J. E. Absorção de nutrientes e distribuição da massa fresca e seca entre órgãos de batata-doce. Horticultura Brasileira, Brasília, v. 27, n. 2, p. 172-178, 2009a.

ECHER, F. R.; DOMINATO, J. C.; CRESTE, J. E.; SANTOS, D. H. Fertilização de cobertura com boro e potássio na nutrição e produtividade da batata-doce. Horticultura Brasileira, Brasília, v. 27, n. 2, p. 167-171, $2009 b$.

GAD, N.; KANDIL, H. Response of sweet potato (Ipomoea batatas L) plants to different levels of cobalt. Australian Journal of Basic and Applied Sciences, Punjab, v. 2, n. 4, p. 949-955, 2008.

GRANBERRY, D. M.; KELLEY, W. T.; BOYHAN, G. Sweet potato: commercial vegetable production. The University of Georgia College of Agricultural and environmental sciences and the U.S. Department of Agriculture cooperating. 2007. 8 p. (Circular, 677).

HARTEMINK, A. E.; POLOMA, S.; MAINO, M.; POWELL, K. S.; EGENAE, J.; O’SULLIVAN, J. N. Yield decline of sweet potato in the humid lowlands of Papua New Guinea. Agriculture, Ecosystems and Environment, Columbus, v. 79, n. 2/3, p. 259-269, 2000.

HOPKINS, B. G.; JOLLEY, V. D.; WEBB, B. L.; ELLSWORTH, J. W.; CALLAHAN, R. K. Boron fertilization in potato. Western Nutrient Management Conference, Salt Lake City, v. 7, p. 215-218, 2007.

MANDAL, M.; ROY, N. R.; MAZUMDAR, D.; DAS, D. $\mathrm{K}$. Effect of different sources of boron on its maintenance in soil in relation to rape (Brassica campestris L.) Archives of Agronomy and Soil Science, v. 55, n. 3, p. 247-253. 2009.

MARCHETTI, M.; MOTOMYA, W.; FABRÍCIO, A.; NOVELINO, J. Resposta do girassol, Helianthus annuus, a fontes e níveis de boro. Acta Scientiarum. Agronomy, Maringá, v. 23, n. 5, p. 1107-1110, 2001.

MESQUiTA, H. A.; ALVARENGA, M. A. R.; PAULA. M. B.; CARVALHO, J. G.; NÓBREGA, J. C. A. Produção e qualidade da batata em resposta ao boro. Ciência $e$ Agrotecnologia, Lavras, v. 31, n. 2, p. 385-392, 2007.

MONTEIRO, D. A.; PERESSIN, V. A. Raízes e tubérculos - batata-doce e cará. In: RAIJ, B. V.; CANTARElla, H.; QUAGGIO, J. A.; FURLANI, A.
M. C. (Ed.). Recomendações de adubação e calagem para o Estado de São Paulo. 2. ed. Campinas: [s.n.], 1997. p. 226. (Boletim técnico, n. 100).

O'SUllivAN, J. N.; ASHER, C. J.; BLAMEY, F. P. C. Nutrient disorders of sweet potato. ACIAR, Monograph $\mathrm{n}^{\circ}$. 48, Australian Centre for International Agricultural Research, Canberra. 1997. 136 p.

PREGNO, L. M.; ARMOUR, J. D. Boron deficiency and toxicity in potato cv. Sebago on oxisol of the Athert Tablelands, North Queensland. Australian Journal of Experimental Agriculture, Austrália, v. 32, n. 2, p. 251253, 1992.

REIS, M. S.; SOUZA, R. J.; CECÍLIO FILHO, A. B. A cultura da batata-doce (Ipomoea batatas (L) LAM). Lavras: UFLA, 1996. 19 p. (Boletim técnico).

SILVA, J. B. C.; LOPES, C. A.; MAGALHÃES, J. S. Cultura da batata-doce. In: CEREDA, M. P. (Ed.). Agricultura: tuberosas amiláceas Latino Americanas. São Paulo: Cargill, 2002. cap. 2, p. 449-503. 
Echer, F. R.; Creste, J. E. 